\title{
RUBIDIUM AND LEAD ABUNDANCES IN GIANT STARS OF THE GLOBULAR CLUSTERS M4 AND M5 ${ }^{1}$
}

\author{
DAVID Yong \\ Research School of Astronomy and Astrophysics, Australian National University, Mount Stromlo Observatory, \\ Cotter Road, Weston Creek, ACT 2611, Australia; yong@mso.anu.edu.au \\ DAVID L. LAMBERT \\ The W. J. McDonald Observatory, University of Texas, Austin, TX 78712; dll@astro.as.utexas.edu \\ Diane B. Paulson \\ NASA Goddard Space Flight Center, Code 693.0, Greenbelt, MD 20771; diane.b.paulson@gsfc.nasa.gov
}

AND

BRUCE W. CARney

Department of Physics and Astronomy, University of North Carolina, Chapel Hill, NC 27599-3255; bruce@physics.unc.edu Received 2007 May 20; accepted 2007 October 9

\begin{abstract}
We present measurements of the neutron-capture elements $\mathrm{Rb}$ and $\mathrm{Pb}$ for bright giants in the globular clusters $\mathrm{M} 4$ and M5. The clusters are of similar metallicity $([\mathrm{Fe} / \mathrm{H}] \simeq-1.2)$, but M4 is decidedly $s$-process enriched relative to $\mathrm{M} 5:[\mathrm{Ba} / \mathrm{Fe}]=+0.6$ for M4 but 0.0 for $\mathrm{M} 5$. The $\mathrm{Rb}$ and $\mathrm{Pb}$ abundances were derived by comparing synthetic spectra with high-resolution, high signal-to-noise ratio spectra obtained with MIKE on the Magellan Telescope. Abundances of $\mathrm{Y}, \mathrm{Zr}$, La, and Eu were also obtained. In M4, the mean abundances from 12 giants are $[\mathrm{Rb} / \mathrm{Fe}]=0.39 \pm 0.02$ $(\sigma=0.07),[\mathrm{Rb} / \mathrm{Zr}]=0.17 \pm 0.03(\sigma=0.08)$, and $[\mathrm{Pb} / \mathrm{Fe}]=0.30 \pm 0.02(\sigma=0.07)$. In M5, the mean abundances from two giants are $[\mathrm{Rb} / \mathrm{Fe}]=0.00 \pm 0.05(\sigma=0.06),[\mathrm{Rb} / \mathrm{Zr}]=0.08 \pm 0.08(\sigma=0.11)$, and $[\mathrm{Pb} / \mathrm{Fe}]=-0.35 \pm$ $0.02(\sigma=0.04)$. Within the measurement uncertainties, the abundance ratios $[\mathrm{Rb} / \mathrm{Fe}],[\mathrm{Pb} / \mathrm{Fe}]$, and $[\mathrm{Rb} / \mathrm{X}]$ for $\mathrm{X}=\mathrm{Y}$, $\mathrm{Zr}$, and $\mathrm{La}$ are constant from star to star in each cluster, and none of these ratios are correlated with $\mathrm{O}$ or $\mathrm{Na}$ abundances. While M4 has a higher $\mathrm{Rb}$ abundance than $\mathrm{M} 5$, the ratios $[\mathrm{Rb} / \mathrm{X}]$ are similar in both clusters, indicating that the nature of the $s$-products is very similar for each cluster but the gas from which M4's stars formed had a higher concentration of these products.
\end{abstract}

Subject headings: Galaxy: abundances - globular clusters: individual (M4, M5) — stars: abundances

Online material: color figures, machine-readable table

\section{INTRODUCTION}

Globular clusters continue to provide a source of fascination and frustration to both theorists and observers. Two notable accomplishments include the use of globular clusters to (1) check the age of the universe (e.g., Gratton et al. 2003) and (2) test and refine our understanding of stellar evolution (e.g., Renzini \& Fusi Pecci 1988). Despite these successes, globular clusters continue to present bewildering puzzles. The most persistent puzzle relates to chemical composition.

For many years, globular clusters have been known to exhibit star-to-star abundance variations for the light elements $\mathrm{C}, \mathrm{N}, \mathrm{O}$, $\mathrm{Na}, \mathrm{Mg}$, and Al (e.g., see reviews by Smith 1987; Kraft 1994; Gratton et al. 2004). While the amplitude of the star-to-star abundance dispersion can vary from cluster to cluster, the now familiar anticorrelations between $\mathrm{C}$ and $\mathrm{N}, \mathrm{O}$ and $\mathrm{Na}$, and $\mathrm{Mg}$ and $\mathrm{Al}$ reveal that the abundance variations are likely produced during hydrogen burning at high temperatures via the $\mathrm{CNO}, \mathrm{Ne}-\mathrm{Na}$, and $\mathrm{Mg}-\mathrm{Al}$ cycles. ( The $\mathrm{O}-\mathrm{Na}$ and $\mathrm{Mg}-\mathrm{Al}$ anticorrelations are not seen in field stars.) However, the stars responsible for the nucleosynthesis and the nature of the pollution mechanism(s) remain poorly understood (see Lattanzio \& Tout 2006 for a recent summary).

One possible explanation for the observed abundance anomalies is internal mixing and nucleosynthesis (e.g., Sweigart \& Mengel 1979; Charbonnel 1995) within the present cluster

\footnotetext{
${ }^{1}$ Based on observations made with the Magellan Clay Telescope at Las Campanas Observatory.
}

members, the so-called evolutionary scenario. The systematic variation of the C, N (Suntzeff \& Smith 1991), and Li (Grundahl et al. 2002) abundances with luminosity along the red giant branch demand an evolutionary component to the star-to-star abundance variations. Dredge-up of $\mathrm{CN}$-cycled material accounts for the $\mathrm{C}$ and $\mathrm{N}$ variations. Development of a giant's convective envelope leading to mixing with highly Li-depleted gas accounts for the decline of the $\mathrm{Li}$ abundance with increasing luminosity. The proton-capture reactions causing the $\mathrm{O}, \mathrm{Na}, \mathrm{Mg}$, and $\mathrm{Al}$ variations demand much higher temperatures and much deeper mixing than those required for $\mathrm{CN}$ cycling. Such mixing is not predicted by standard theoretical models of red giants, and the discovery of the $\mathrm{O}, \mathrm{Na}, \mathrm{Mg}$, and $\mathrm{Al}$ anomalies in main-sequence stars (e.g., Briley et al. 1996; Gratton et al. 2001) eliminates deep mixing as a viable explanation for the $\mathrm{O}-\mathrm{Al}$ variations. The interiors of main-sequence stars are too cool to process $\mathrm{Ne}$ to $\mathrm{Na}$ or $\mathrm{Mg}$ to Al. Therefore, the cluster gas must have been inhomogeneous when the present stars were formed. This alternative explanation for the abundance anomalies is the so-called primordial scenario.

In the primordial scenario, intermediate-mass asymptotic giant branch stars (IM-AGBs) from the generation to which the observed stars belong have long been considered candidates for synthesizing the abundance variations (Cottrell \& Da Costa 1981). In IM-AGBs, the convective envelope can reach the top of the hydrogen-burning shell, a process called hot-bottom burning. For sufficiently massive and metal-poor AGBs, the temperatures at the base of the convective envelope can exceed 100 million degrees, thereby allowing the efficient operation of the $\mathrm{CNO}, \mathrm{Ne}-\mathrm{Na}$, and 
Mg-Al cycles (e.g., Karakas \& Lattanzio 2003). That IM-AGBs do not alter the abundances of the alpha or iron-peak elements (as required by observations) adds to their qualitative appeal. However, quantitative tests reveal problems with the IM-AGB primordial scenario. Theoretical yields from IM-AGBs combined with a chemical evolution model (Fenner et al. 2004) suggest that $\mathrm{O}$ is not sufficiently depleted, $\mathrm{Na}$ is overproduced, $\mathrm{Mg}$ is produced rather than destroyed, the isotope ratios of $\mathrm{Mg}$ do not match the observations, and the sum of $\mathrm{C}+\mathrm{N}+\mathrm{O}$ increases substantially in contrast to the observations. Ventura \& D'Antona (2005a, 2005b, 2005c) find that many of the flaws noted above can be alleviated when IM-AGB yields are calculated using a revised treatment for convection and mass loss. However, Ventura \& D'Antona note that problems persist, namely, with the $\mathrm{Mg}$ isotope ratios, and warn that the predictive power of the current AGB models is limited. Recently, Prantzos \& Charbonnel (2006), Smith (2006), and Decressin et al. (2007) suggested that the winds from massive stars may be more promising candidates than IM-AGBs. There is no satisfactory explanation for the complex patterns for the light-element abundances exhibited by every well-studied Galactic globular cluster. Therefore, our present understanding of globular cluster chemical evolution and/or stellar nucleosynthesis is incomplete.

Determinations of the stellar abundances of the transiron or heavy elements offer clues to the history behind the chemical evolution of globular clusters. Here, we provide novel informationthe $\mathrm{Rb}$ and $\mathrm{Pb}$ abundances - for giants in M4 and M5, a pair of clusters of similar metallicity but with distinctly different levels of $s$-process products. The quintessential $r$-process element $\mathrm{Eu}$ has similar abundances in the two clusters and, indeed, across the collection of Galactic globular clusters. In sharp contrast, the $s$-process products are more evident in M4 than in M5 and other clusters of similar metallicity: $[\mathrm{Ba} / \mathrm{Fe}]$ is about +0.6 in $\mathrm{M} 4$ but 0.0 in M5. The questions - are there differences in the $\mathrm{Rb}$ and $\mathrm{Pb}$ abundances between this pair of clusters, and are the star-to-star variations in the abundances of light elements $(\mathrm{O}, \mathrm{Na}, \mathrm{Mg}$, and $\mathrm{Al})$ reflected in variations among the abundances of $\mathrm{Rb}$ and $\mathrm{Pb}$ ? - seem likely to probe the origins of the $s$ - and $r$-process products for globular clusters.

Due to a critical branching point in the $s$-process path at ${ }^{85} \mathrm{Kr}$, the abundance of $\mathrm{Rb}$ relative to $\mathrm{Sr}, \mathrm{Y}$, or $\mathrm{Zr}$ can differ by a factor of 10 depending on the neutron density at the $s$-process site. In the case of AGB stars, the neutron density in the He shell is dependent on the stellar mass (e.g., see Tomkin \& Lambert 1983; Lambert et al. 1995; Busso et al. 1999; Abia et al. 2001 for further details). Since the isotopes of $\mathrm{Pb}$ and $\mathrm{Bi}$ are the last stable nuclei on the $s$-process path, the $s$-process terminates at these elements and overabundances of $\mathrm{Pb}$ and $\mathrm{Bi}$ will arise if seed nuclei are shuffled by neutron captures down the entire $s$-process path. In particular, metal-poor AGB stars may produce large overabundances of $\mathrm{Pb}$ and $\mathrm{Bi}$ if the neutron supply per seed exceeds a critical value (e.g., see Goriely \& Siess 2001; Travaglio et al. 2001; Busso et al. 2001 for further details). The suspicion is that the star-to-star abundance variations for light elements are due to contamination by IM-AGBs. Some contend that IM-AGBs also synthesize $s$-process nuclides, and then one might expect to see star-to-star variations in the $\mathrm{Rb}$ and $\mathrm{Pb}$ abundances, as well as correlations with light-element abundances.

To further examine the possible role of IM-AGBs in the chemical evolution of globular clusters, Yong et al. (2006b) measured $\mathrm{Rb}$ and $\mathrm{Pb}$ in NGC 6752 and M13, the two clusters that exhibit the largest amplitude for Al variations. It was found that the abundance ratios $[\mathrm{Rb} / \mathrm{Zr}]$ and $[\mathrm{Pb} / \mathrm{Fe}]$ were constant from star to star within the measurement uncertainties. If IM-AGBs do synthesize
$\mathrm{Rb}$ and $\mathrm{Pb}$, then they may not be responsible for the abundance variations. On the other hand, if IM-AGBs are responsible for the abundance variations, they cannot synthesize $\mathrm{Rb}$ or $\mathrm{Pb}$.

In this paper, we extend the measurements of $\mathrm{Rb}$ and $\mathrm{Pb}$ to the globular clusters M4 and M5. While these clusters are more metal-rich than NGC 6752 or M13, both M4 and M5 are known to exhibit large dispersions and correlations for the light-element abundances (see pioneering studies on $\mathrm{CN}$ bimodality by Norris [1981] and Smith \& Norris [1983], as well as recent high-resolution spectroscopic studies by Ivans et al. [1999, 2001], Ramírez \& Cohen [2003], and references therein). In particular, as noted above, M4 is remarkably, perhaps uniquely among globular clusters, enriched in $s$-process products.

\section{OBSERVATIONS AND DATA REDUCTION}

The targets included 12 stars in M4 previously studied by Ivans et al. (1999) and two stars in M5 previously studied by Ivans et al. (2001) and Ramírez \& Cohen (2003). The main focus was to observe a large number of stars in M4, and although we were restricted to the brightest giants, we note that the sample spans a considerable range of the known star-to-star elemental abundance variations. The smaller sample in M5 is due to the fact that those observations were conducted during other observing programs when primary targets were unavailable. In Table 1 we list the program stars. The stellar identifications for M4 and M5 are from Lee (1977) and Arp (1962), respectively.

The observations were performed with the Magellan Telescope using the Magellan Inamori Kyocera Echelle spectrograph (MIKE; Bernstein et al. 2003) on 2004 June 12-13, July 16, and July 18 . A $0.35^{\prime \prime}$ slit was used providing a resolving power of $R \equiv \lambda / \Delta \lambda=55,000$ in the red and $R=65,000$ in the blue per 4 pixel resolution element with wavelength coverage from 3800 to $8500 \AA$. The IRAF ${ }^{2}$ package of programs was used for most of the data reduction. In order to correct for the fact that the lines are severely tilted with respect to the orders and the tilt varies across the CCD, we used the MTOOLS ${ }^{3}$ set of tasks written by J. Baldwin to extract the spectral orders. In the one-dimensional wavelength-calibrated normalized spectra, the typical signal-tonoise ratio was $70 \mathrm{pixel}^{-1}$ at $4050 \AA$ (140 per resolution element) and 400 pixel $^{-1}$ at $7800 \AA$ (800 per resolution element).

\section{ANALYSIS}

\subsection{Stellar Parameters and the Iron Abundance}

The required stellar parameters for an abundance analysis are the effective temperature $\left(T_{\text {eff }}\right)$, the surface gravity $(\log g)$, and the microturbulent velocity $\left(\xi_{t}\right)$. Our analysis techniques closely follow Yong et al. (2006b), in which we determined these values adopting a traditional spectroscopic approach. Using routines in IRAF, we measured the equivalent widths (EWs) for a set of $\mathrm{Fe}_{\mathrm{I}}$ and $\mathrm{Fe}$ II lines. The set of Fe lines was identical to those used by Yong et al. (2006a) for which the $g f$-values were taken from Lambert et al. (1996), Biémont et al. (1991), Blackwell et al. (1995), and references therein. Model atmospheres were taken from the Kurucz (1993) local thermodynamic equilibrium (LTE) stellar atmosphere grid, and we interpolated within the grid when necessary. We used the LTE stellar line analysis program MOOG (Sneden 1973) to determine abundances for a given line. We adjusted $T_{\text {eff }}$ until

\footnotetext{
2 IRAF (Image Reduction and Analysis Facility) is distributed by the National Optical Astronomy Observatory, which is operated by the Association of Universities for Research in Astronomy, Inc., under contract with the National Science Foundation.

3 See http://www.lco.cl/telescopes-information/magellan/instruments-1/mike/ IRAF_tools/iraf-mtools-package/iraf-mtools-package.
} 
TABLE 1

Program Stars, Exposure Times, and Stellar Parameters

\begin{tabular}{|c|c|c|c|c|c|c|c|}
\hline \multirow[b]{2}{*}{ StAR } & \multirow{2}{*}{$\begin{array}{l}\text { EXPOSURE TIME } \\
\text { (minutes) }\end{array}$} & \multicolumn{2}{|c|}{$\mathrm{S} / \mathrm{N}^{\mathrm{a}}$} & \multirow{2}{*}{$\begin{array}{l}T_{\text {eff }} \\
(\mathrm{K})\end{array}$} & \multirow[b]{2}{*}{$\log g$} & \multirow{2}{*}{$\begin{array}{c}\xi_{t} \\
\left(\mathrm{~km} \mathrm{~s}^{-1}\right)\end{array}$} & \multirow[b]{2}{*}[\mathrm{Fe}/\mathrm{H}]{} \\
\hline & & $4050 \AA ̊$ & $7800 \AA$ & & & & \\
\hline M4 L1411 .............. & 40 & 61 & 397 & 4025 & 0.80 & 1.75 & -1.23 \\
\hline M4 L1501 ............... & 151 & 72 & 353 & 4175 & 1.00 & 1.55 & -1.29 \\
\hline M4 L1514 .............. & 35 & 59 & 432 & 3950 & 0.30 & 1.85 & -1.22 \\
\hline M4 L2307 .............. & 80 & 82 & 453 & 4125 & 0.95 & 1.65 & -1.19 \\
\hline M4 L2406 ............... & 30 & 72 & 395 & 4150 & 0.15 & 2.20 & -1.30 \\
\hline M4 L2617 ............... & 140 & 71 & 377 & 4275 & 1.25 & 1.65 & -1.20 \\
\hline M4 L3209 ............... & 35 & 68 & 407 & 4075 & 0.75 & 1.95 & -1.25 \\
\hline M4 L3413 ............. & 135 & 88 & 440 & 4225 & 1.10 & 1.75 & -1.23 \\
\hline M4 L3624 ............... & 80 & 39 & 285 & 4225 & 1.05 & 1.60 & -1.29 \\
\hline M4 L4511 ............... & 80 & 75 & 376 & 4150 & 1.05 & 1.70 & -1.22 \\
\hline M4 L4611 ............... & 60 & 29 & 393 & 3925 & 0.15 & 1.45 & -1.09 \\
\hline M4 L4613 .............. & 45 & 42 & 420 & 3900 & 0.20 & 1.70 & -1.25 \\
\hline M5 IV-81 ................. & 77 & 25 & 210 & 4050 & 0.30 & 1.90 & -1.28 \\
\hline M5 IV-82 ................. & 190 & 79 & 218 & 4400 & 1.20 & 1.75 & -1.33 \\
\hline
\end{tabular}

${ }^{a} \mathrm{~S} / \mathrm{N}$ values are per pixel.

there was no trend between the abundances from $\mathrm{Fe}_{\mathrm{I}}$ lines and the lower excitation potential. We adjusted $\log g$ until the abundances from $\mathrm{Fe}$ I and $\mathrm{Fe}$ II lines were in agreement. We adjusted $\xi_{t}$ until there was no trend between the abundances from $\mathrm{Fe}_{\mathrm{I}}$ lines and $\mathrm{EW}$. This process was iterated until all three parameters were simultaneously constrained. The final $[\mathrm{Fe} / \mathrm{H}]$ was the mean from all $\mathrm{Fe}$ lines assuming a solar abundance $\log \epsilon(\mathrm{Fe})=7.50$. The stellar parameters are given in Table 1, and we estimate the internal errors to be $T_{\text {eff }} \pm 50 \mathrm{~K}, \log g \pm 0.2 \mathrm{dex}$, and $\xi_{t} \pm 0.2 \mathrm{~km} \mathrm{~s}^{-1}$.

As an additional check on our surface gravities (and analysis techniques), we compared our $\log g$ values with the $Y^{2}$ isochrones (Demarque et al. 2004). We adopted the $13 \mathrm{Gyr}$ isochrones with $[\alpha / \mathrm{Fe}]=+0.3$, our spectroscopic $T_{\text {eff }}$, and interpolated between the two closest metallicities, $z=0.004$ and 0.001 . We found that our surface gravities were in fair agreement with the $Y^{2}$ isochrones, $\log g_{\text {spec }}-\log g_{\text {isochrone }}=-0.22 \pm 0.07(\sigma=0.26)$.

The stellar parameters are in good agreement with those obtained by Ivans et al. $(1999,2001)$ and Ramírez \& Cohen $(2003)$. For the 12 M4 giants, the mean differences (this study - Ivans et al. 1999) are $\Delta T_{\text {eff }}=71 \pm 17(\sigma=58 \mathrm{~K}), \Delta \log g=0.02 \pm$ $0.05(\sigma=0.17), \Delta \xi_{t}=0.04 \pm 0.04\left(\sigma=0.15 \mathrm{~km} \mathrm{~s}^{-1}\right)$, and $\Delta[\mathrm{Fe} / \mathrm{H}]=-0.05 \pm 0.01(\sigma=0.05)$. For the two M5 giants, the mean differences (this study - Ramírez \& Cohen 2003) are $\Delta T_{\text {eff }}=20 \pm 5(\sigma=7 \mathrm{~K}), \Delta \log g=-0.15 \pm 0.15(\sigma=0.21)$, $\Delta \xi_{t}=-0.16 \pm 0.03\left(\sigma=0.04 \mathrm{~km} \mathrm{~s}^{-1}\right)$, and $\Delta[\mathrm{Fe} / \mathrm{H}]=0.05 \pm$ $0.02(\sigma=0.03)$. For M5 IV-81, our $T_{\text {eff }}$ is $105 \mathrm{~K}$ higher, $\log g$ is 0.3 dex higher, $\xi_{t}$ is $0.02 \mathrm{~km} \mathrm{~s}^{-1}$ lower, and $[\mathrm{Fe} / \mathrm{H}]$ is $0.12 \mathrm{dex}$ higher than the values determined by Ivans et al. (2001).

\subsection{Rubidium Abundances}

The abundances for $\mathrm{Rb}$ were determined via spectrum synthesis of the $7800 \AA \mathrm{Rb}_{\mathrm{I}}$ line (see Fig. 1). For all stars in our sample, this $\mathrm{Rb}$ line is blended with a $\mathrm{Si}$ I line, as well as with weak CN lines, and therefore an equivalent width analysis is not possible. Even in the coolest stars, the $\mathrm{Rb}$ line is only $10 \%$ deep relative to the continuum, such that accurate abundances can be derived only from high-resolution, high signal-to-noise ratio spectra. The adopted wavelengths and relative strengths for the isotopic and hyperfine-structure components of $\mathrm{Rb}$ were identical to Lambert \& Luck (1976) and Tomkin \& Lambert (1999). We assumed a solar isotope ratio ${ }^{85} \mathrm{Rb} /{ }^{87} \mathrm{Rb}=3$, and the macroturbulent broadening was fixed by fitting the profile of the nearby $7798 \AA$
$\mathrm{Ni}$ I line. Synthetic spectra were generated using MOOG, and the $\mathrm{Si}$ and $\mathrm{Rb}$ abundances were varied to obtain the best fit to the observed spectrum. Our tests confirmed the finding by Lambert \& Luck (1976) that $\mathrm{Rb}$ isotope ratios cannot be measured from the $7800 \AA$ line due to the hyperfine structure and the small isotopic shift. Not surprisingly, our tests showed that the derived

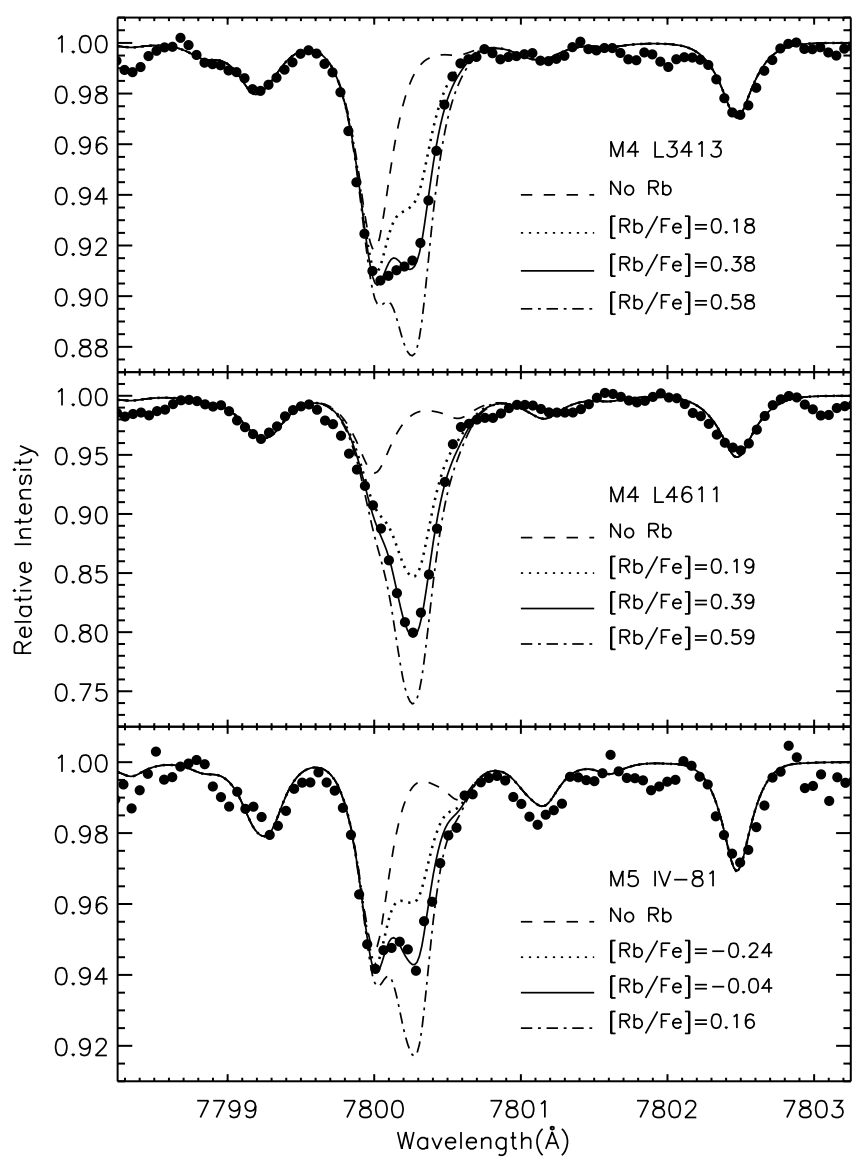

FIG. 1.-Observed spectra ( filled circles) for M4 L3413 (top), M4 L4611 (middle), and M5 IV-81 (bottom) near the $7800 \AA$ Rb feature. Synthetic spectra with different $\mathrm{Rb}$ abundances are shown (the solid line represents the best fit). [See the electronic edition of the Journal for a color version of this figure.] 


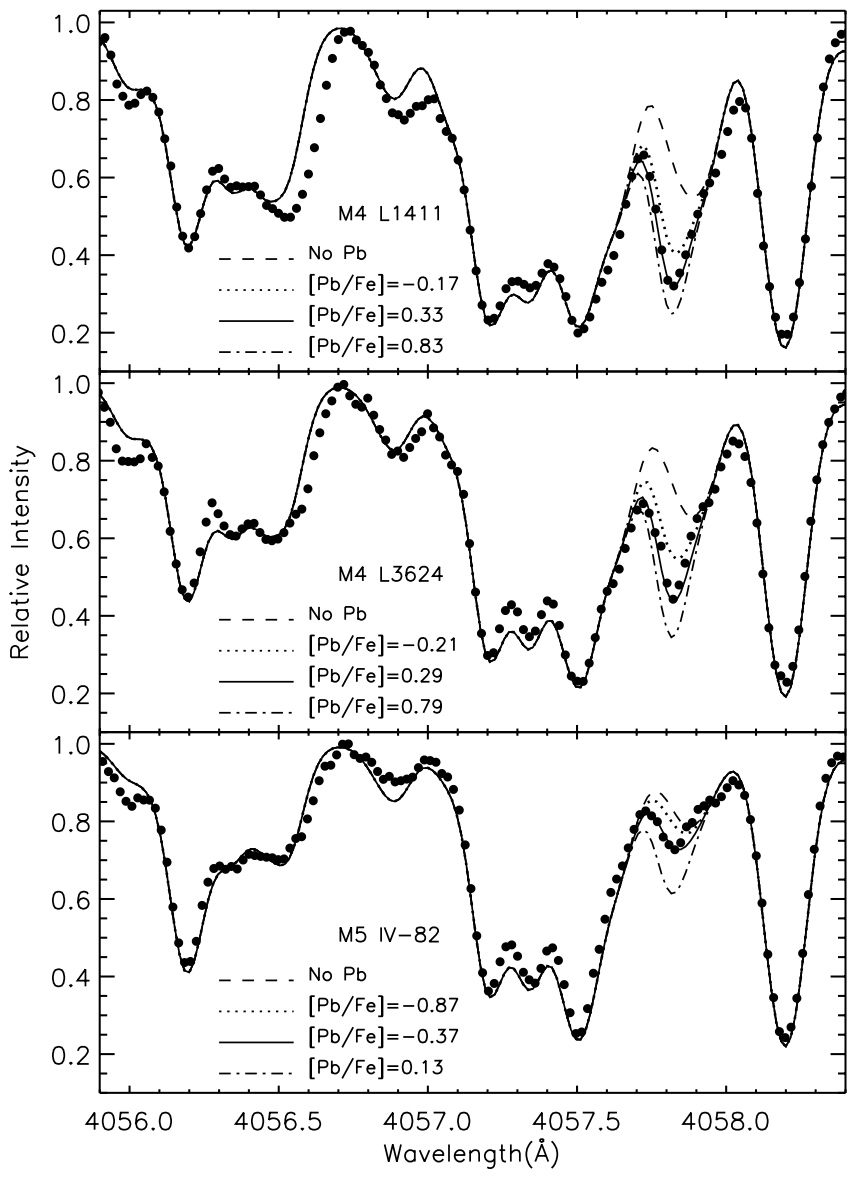

Fig. 2.-Observed spectra ( filled circles) for M4 L1411 (top), M4 L3624 (middle), and M5 IV-82 (bottom) near the $4058 \AA \mathrm{Pb}$ feature. Synthetic spectra with different $\mathrm{Pb}$ abundances are shown (the solid line represents the best fit). [See the electronic edition of the Journal for a color version of this figure.]

$\mathrm{Rb}$ abundances are insensitive to the assumed isotope ratio. In Yong et al. (2006b) we took the Kurucz et al. (1984) solar atlas and measured an abundance $\log \epsilon(\mathrm{Rb})=2.58$ using a model atmosphere with $T_{\text {eff }} / \log g / \xi_{t}=5770 / 4.44 / 0.85$. Our solar Rb abundance is in excellent agreement with the Grevesse \& Sauval (1998) value, $\log \epsilon(\mathrm{Rb})=2.60$. The weaker $\mathrm{Rb}$ I resonance line near $7947 \AA$ is detected but in a region affected by unidentified blends, atmospheric absorption, and fringing. While preliminary analyses suggest that the abundances derived from the $7947 \AA$ line are similar to those derived from the $7800 \AA$ line (see Yong et al. [2006b] for a more detailed comparison), we restrict our analyses to the $7800 \AA$ line.

In this study, we included $\mathrm{CN}$ molecular lines when fitting the $7800 \AA \mathrm{Rb}$ line. The $\mathrm{CN}$ lines were taken from R. Kurucz's list. ${ }^{4}$ While the inclusion of these lines resulted in an improved fit to the local continuum, we note that the $\mathrm{Rb}$ abundances were unaffected. Even for the coolest stars in M4 for which the CN line strength was at a maximum, the $\mathrm{Rb}$ abundances increased by $\leq 0.02 \mathrm{dex}$ if the $\mathrm{CN}$ lines were omitted. For the more metal-poor cluster NGC 6752, we reanalyzed a subset of the Yong et al. (2006b) spectra and found that the $\mathrm{Rb}$ abundances were unchanged when $\mathrm{CN}$ lines were included in the analysis.

\subsection{Lead Abundances}

The abundances for $\mathrm{Pb}$ were determined via spectrum synthesis of the $4058 \AA \mathrm{Pb}$ I line (see Fig. 2). High-resolution, high

\footnotetext{
${ }^{4}$ See http://kurucz.harvard.edu/LINELISTS/LINESMOL/.
}

signal-to-noise ratio spectra are essential for measuring $\mathrm{Pb}$ abundances because the region near $4058 \AA$ is crowded with molecular lines of $\mathrm{CH}$ as well as $\mathrm{Mg}$, $\mathrm{Ti}, \mathrm{Mn}, \mathrm{Fe}$, and $\mathrm{Co}$ atomic lines. Since M4 and M5 are more metal-rich than NGC 6752 and M13, the $4058 \AA$ region is more crowded and the uncertainties in the derived $\mathrm{Pb}$ abundances are greater. However, we note that our syntheses provide a very good fit to the observed spectra. The macroturbulent broadening was fixed by fitting the profiles of nearby lines. The $g f$-value of the $\mathrm{Pb}$ I line was the same as that used by Aoki et al. (2002), as were the hyperfine-structure and isotopic components. We assumed a solar isotope ratio, and our tests confirmed that the derived $\mathrm{Pb}$ abundances do not depend on the assumed isotope ratio. For the solar $\mathrm{Pb}$ abundance, we adopted $\log \epsilon(\mathrm{Pb})=1.95$ from Grevesse \& Sauval (1998).

\subsection{Additional Elements}

The abundances for $\mathrm{O}, \mathrm{Na}, \mathrm{Mg}, \mathrm{Al}, \mathrm{Y}, \mathrm{Zr}, \mathrm{La}$, and Eu were also measured in the program stars using the same lines as Yong et al. (2005). Y and Zr were selected since the ratios $[\mathrm{Rb} / \mathrm{Y}]$ and $[\mathrm{Rb} / \mathrm{Zr}]$ are sensitive to the neutron density at the site of the $s$-process. $\mathrm{O}$, $\mathrm{Na}, \mathrm{Mg}$, and $\mathrm{Al}$ were measured since they are known to vary from star to star in these and other globular clusters. Remeasuring these elements in M4 and M5 provides a check to see if our abundance determinations are on the same scale as other investigators. Similarly, the neutron-capture elements $\mathrm{La}$ and Eu were measured (hyperfine and/or isotopic splitting was included). When deriving $\mathrm{O}$ abundances, we generated synthetic spectra to account for possible blending from $\mathrm{CN}$ molecular lines. We assumed $\mathrm{C}$ and $\mathrm{N}$ abundances interpolated as a function of $\mathrm{Na}$ with the $\mathrm{C}$ and $\mathrm{N}$ abundances taken from Ivans et al. $(1999,2001)$ and Smith et al. (1997). A subset of abundance determinations were conducted using spectrum synthesis to account for possible blends. In Table 2 we present the measured elemental abundances for $\mathrm{O}, \mathrm{Na}, \mathrm{Mg}, \mathrm{Al}$, $\mathrm{Rb}, \mathrm{Y}, \mathrm{Zr}, \mathrm{La}, \mathrm{Eu}$, and $\mathrm{Pb}$. The adopted solar abundances were $8.69,6.33,7.58,6.47,2.60,2.24,2.60,1.13,0.52$, and 1.95, respectively. These values are identical to those we used in previous papers and were taken from Grevesse \& Sauval (1998) for all elements except $\mathrm{O}$ (Allende Prieto et al. 2001), La (Lawler et al. 2001a), and Eu (Lawler et al. 2001b). Table 2 also contains the $\mathrm{Zr}$ abundances shifted onto the Smith et al. (2000) scale.

For M4, the measured elemental abundances are in good agreement with Ivans et al. (1999). The mean differences (this study - Ivans et al. 1999) are $\Delta[\mathrm{O} / \mathrm{Fe}]=0.34 \pm 0.03(\sigma=0.11)$, $\Delta[\mathrm{Na} / \mathrm{Fe}]=0.14 \pm 0.03(\sigma=0.12), \Delta[\mathrm{Mg} / \mathrm{Fe}]=0.14 \pm 0.02$ $(\sigma=0.06), \Delta[\mathrm{Al} / \mathrm{Fe}]=0.05 \pm 0.03(\sigma=0.10), \Delta[\mathrm{La} / \mathrm{Fe}]=$ $0.01 \pm 0.03(\sigma=0.10)$, and $\Delta[\mathrm{Eu} / \mathrm{Fe}]=0.03 \pm 0.02(\sigma=$ $0.07)$. The agreement for $\mathrm{Al}, \mathrm{La}$, and $\mathrm{Eu}$ is excellent. The largest discrepancy is for $\mathrm{O}$, and differences in the adopted solar abundances can account for 0.24 dex leaving a 0.10 dex residual. While both studies use the same $\mathrm{O}$ lines and $g f$ values, they employ spectrum synthesis, which presumably involves a different set of atomic and molecular lines. A small offset also exists for $\mathrm{Na}$ and $\mathrm{Mg}$, which could be due to the different set of lines employed.

For M5, the elemental abundances are in fair agreement with Ramírez \& Cohen (2003). The mean differences (this study Ramírez \& Cohen 2003) are $\Delta[\mathrm{O} / \mathrm{Fe}]=0.27 \pm 0.14(\sigma=0.20)$, $\Delta[\mathrm{Na} / \mathrm{Fe}]=-0.05 \pm 0.10(\sigma=0.14), \Delta[\mathrm{Mg} / \mathrm{Fe}]=0.11 \pm 0.06$ $(\sigma=0.08), \Delta[\mathrm{Zr} / \mathrm{Fe}]=-0.05 \pm 0.19(\sigma=0.27), \Delta[\mathrm{La} / \mathrm{Fe}]=$ $-0.03 \pm 0.03(\sigma=0.04)$, and $\Delta[\mathrm{Eu} / \mathrm{Fe}]=-0.06 \pm 0.09(\sigma=$ 0.13 ). For $O$, the difference can be attributed to the adopted solar abundance. For the single star also studied by Ivans et al. (2001) the measured abundances are in good agreement $\Delta[\mathrm{X} / \mathrm{Fe}] \leq$ 0.07 dex. The difference for $\mathrm{La}$ is $0.14 \mathrm{dex}$ (possibly due to the different set of lines) and 0.58 dex for $O$. The adopted solar 
TABLE 2

Elemental Abundances

\begin{tabular}{|c|c|c|c|c|c|c|c|c|c|c|c|}
\hline Star & {$[\mathrm{O} / \mathrm{Fe}]$} & {$[\mathrm{Na} / \mathrm{Fe}]$} & {$[\mathrm{Mg} / \mathrm{Fe}]$} & {$[\mathrm{Al} / \mathrm{Fe}]$} & {$[\mathrm{Rb} / \mathrm{Fe}]$} & {$[\mathrm{Y} / \mathrm{Fe}]$} & {$[\mathrm{Zr} / \mathrm{Fe}]^{\mathrm{a}}$} & {$[\mathrm{Zr} / \mathrm{Fe}]^{\mathrm{b}}$} & {$[\mathrm{La} / \mathrm{Fe}]$} & {$[\mathrm{Eu} / \mathrm{Fe}]$} & {$[\mathrm{Pb} / \mathrm{Fe}]$} \\
\hline M4 L1411 ................... & 0.46 & 0.55 & 0.55 & 0.80 & 0.45 & 0.74 & 0.62 & 0.32 & 0.58 & 0.49 & 0.33 \\
\hline M4 L1501 ................. & 0.57 & 0.51 & 0.59 & 0.79 & 0.37 & 0.69 & 0.43 & 0.13 & 0.48 & 0.47 & 0.29 \\
\hline M4 L1514 ................. & 0.69 & 0.10 & 0.50 & 0.63 & 0.32 & 0.71 & 0.62 & 0.32 & 0.28 & 0.25 & 0.17 \\
\hline M4 L2307 ................. & 0.47 & 0.50 & 0.54 & 0.76 & 0.44 & 0.79 & 0.53 & 0.23 & 0.49 & 0.46 & 0.34 \\
\hline M4 L2406 ............... & 0.51 & 0.46 & 0.58 & 0.66 & 0.25 & $\ldots$ & $\ldots$ & $\ldots$ & 0.28 & 0.18 & 0.20 \\
\hline M4 L2617 ................. & 0.44 & 0.48 & 0.52 & 0.76 & 0.39 & 0.63 & 0.51 & 0.21 & 0.51 & 0.44 & 0.25 \\
\hline M4 L3209 ................. & 0.67 & 0.42 & 0.60 & 0.75 & 0.49 & 0.67 & 0.65 & 0.35 & 0.53 & 0.44 & 0.35 \\
\hline M4 L3413 ................. & 0.76 & 0.06 & 0.57 & 0.67 & 0.38 & 0.62 & 0.51 & 0.21 & 0.49 & 0.44 & 0.33 \\
\hline M4 L3624 .................. & 0.78 & 0.18 & 0.64 & 0.66 & 0.40 & 0.68 & 0.49 & 0.19 & 0.49 & 0.41 & 0.29 \\
\hline M4 L4511 ................ & 0.49 & 0.50 & 0.60 & 0.76 & 0.37 & 0.70 & 0.42 & 0.12 & 0.51 & 0.48 & 0.27 \\
\hline M4 L4611 ................. & 0.25 & 0.67 & 0.47 & 0.75 & 0.39 & $\ldots$ & $\ldots$ & $\ldots$ & 0.51 & 0.29 & 0.34 \\
\hline M4 L4613 ................. & 0.59 & 0.67 & 0.62 & 0.86 & 0.47 & $\ldots$ & $\ldots$ & $\ldots$ & 0.56 & 0.39 & 0.45 \\
\hline M5 IV-81 .................. & 0.50 & 0.15 & 0.35 & 0.33 & -0.04 & 0.22 & 0.25 & -0.05 & 0.17 & 0.46 & -0.32 \\
\hline M5 IV-82 ................ & 0.69 & -0.22 & 0.40 & 0.06 & 0.05 & 0.12 & 0.19 & -0.11 & 0.22 & 0.59 & -0.37 \\
\hline
\end{tabular}

a $\mathrm{Zr}$ abundances when using the $g f$ values and solar abundance adopted in Yong et al. (2005).

b $\mathrm{Zr}$ abundances when shifted onto the Smith et al. (2000) scale_-see Yong et al. (2006b) for details.

abundances can account for 0.24 dex leaving a 0.34 dex residual. I. Ivans kindly sent the synthetic and observed spectra near the 6300 and $6363 \AA$ lines used to derive $\mathrm{O}$ abundances. The difference in the measured $\mathrm{O}$ abundances between the two studies is $\Delta \log \epsilon(\mathrm{O})=0.14 \mathrm{dex}$. The difference in the measured Fe abundance is 0.12 dex. The remaining 0.08 dex residual may be attributed to differences in the spectrum synthesis analysis.

The adopted line lists and measured equivalent widths are presented in Table 3. In Table 4 the abundance dependences on the model parameters are given.

\section{DISCUSSION}

\subsection{The Heavy-Element Canvas}

The heavy elements are synthesized by neutron-captures in the $s$ - and $r$-processes. (We ignore here the nuclides, all of low abundance, referred to as $p$-nuclides.) In all but material dominated by $s$-processed products, Eu is a signature element for the $r$-process. There is evidence from analyses of field stars, especially those severely enriched in $r$-process products that the relative abundances for the $r$-process of nuclides from $\mathrm{Ba}$ to Eu are invariant with metallicity (e.g., see Cowan \& Sneden 2006). This invariance does not extend to the ratio of heavy products (i.e., Eu) to light products (i.e., Sr, Y, and Zr). In a solar mix of elements, Ba is taken as a measure of the $s$-process with but a slight contamination from the $r$-process (Burris et al. 2000). The [Ba/Eu] ratio of a star is widely taken to indicate the relative mix of $s$ - to $r$-processed material.
In the case of ratios such as $[\mathrm{Eu} / \mathrm{Fe}]$ and $[\mathrm{Ba} / \mathrm{Fe}]$ for globular cluster stars, three questions arise: Is there an intercluster spread in the ratios? Is there an intracluster spread, especially for clusters exhibiting a large spread among light element abundances? How do the cluster ratios compare with those found for the field stars, as a function of $[\mathrm{Fe} / \mathrm{H}]$ ?

\subsubsection{The $[\mathrm{Eu} / \mathrm{Fe}]$ Ratio}

With but the single known exception of Ruprecht 106, the $[\mathrm{Eu} / \mathrm{Fe}]$ ratio across the collection of examined Galactic globular clusters with $[\mathrm{Fe} / \mathrm{H}]$ from about -1.0 to -2.5 appears to be singlevalued. Values of $[\mathrm{Eu} / \mathrm{Fe}]$ in the literature for well-sampled clusters with $[\mathrm{Fe} / \mathrm{H}]<-1$ span a reported range of only 0.2 dex from about +0.35 to +0.55 with no obvious trend with $[\mathrm{Fe} / \mathrm{H}]$. (Rup 106 is an outlier at 0.0 from analyses of two stars [Brown et al. 1997]. M80 is probably also an outlier at +1.0 , but the analyses were based on less than ideal spectra, $R=18,000$ [Cavallo et al. 2004].) This range is gathered from examination of the extensive literature; references cited by Gratton et al. (2004) were read and supplemented by more recent papers. No attempt has been made by us to correct published values to a common standard. It would be premature to claim that the 0.2 dex spread in $[\mathrm{Eu} / \mathrm{Fe}]$ is real and thus not entirely attributable to cumulative errors of measurement. Our results $[\mathrm{Eu} / \mathrm{Fe}]=+0.41$ for $\mathrm{M} 4$ and +0.53 for M5 fall within this range and, as noted above, are consistent with previously published results.

Although our samples are small (see also Yong et al. 2006b), the stars chosen for their contrasting light-element abundances

TABLE 3

Line Lists and Equivalent Widths for Program Stars

\begin{tabular}{|c|c|c|c|c|c|c|c|c|c|c|c|c|c|c|c|c|c|}
\hline $6300.30 \ldots \ldots \ldots$ & 8.0 & 0.00 & -9.75 & \multicolumn{14}{|c|}{ Abundances derived using synthetic spectra } \\
\hline $4982.83 \ldots \ldots \ldots$ & 11.0 & 2.10 & -0.91 & $\cdots$ & 93.4 & .. & 122.5 & $\ldots$ & 92.2 & $\ldots$ & $\ldots$ & 69.5 & 104.7 & $\cdots$ & 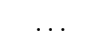 & 74.4 & 29.7 \\
\hline $5682.65 \ldots \ldots \ldots . . .$. & 11.0 & 2.10 & -0.71 & $\ldots$ & 117.9 & 119.4 & $\ldots$ & $\ldots$ & 121.1 & $\ldots$ & 96.1 & 96.8 & $\ldots$ & $\ldots$ & $\ldots$ & 102.8 & 51.0 \\
\hline $5688.22 \ldots \ldots \ldots . . . .$. & 11.0 & 2.10 & -0.40 & $\ldots$ & $\ldots$ & $\ldots$ & $\ldots$ & $\ldots$ & $\ldots$ & $\ldots$ & 114.7 & 114.7 & $\ldots$ & $\ldots$ & $\ldots$ & $\ldots$ & 69.6 \\
\hline
\end{tabular}

Notes.-Table 3 is published in its entirety in the electronic edition of the Astrophysical Journal. A portion is shown here for guidance regarding its form and content. 
TABLE 4

Abundance Dependences on Model Parameters for M4 L2307

\begin{tabular}{|c|c|c|c|}
\hline Species & $T_{\text {eff }}+50$ & $\log g+0.2$ & $\xi_{t}+0.2$ \\
\hline$[\mathrm{Fe} / \mathrm{H}] \ldots \ldots \ldots \ldots \ldots \ldots \ldots \ldots \ldots$ & -0.01 & 0.04 & -0.03 \\
\hline 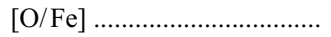 & 0.01 & 0.02 & 0.02 \\
\hline$[\mathrm{Na} / \mathrm{Fe}] \ldots$. & 0.06 & -0.07 & 0.01 \\
\hline 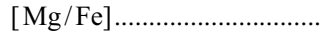 & 0.04 & -0.05 & 0.02 \\
\hline (n.................... & 0.05 & -0.06 & 0.02 \\
\hline$[\mathrm{Rb} / \mathrm{Fe}] \ldots \ldots \ldots \ldots \ldots \ldots \ldots \ldots \ldots \ldots \ldots \ldots \ldots \ldots \ldots \ldots \ldots \ldots$ & 0.08 & -0.05 & 0.03 \\
\hline$[\mathrm{Y} / \mathrm{Fe}] .$. & 0.00 & 0.03 & -0.02 \\
\hline 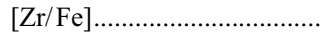 & 0.13 & -0.03 & 0.02 \\
\hline (n) & 0.02 & 0.02 & 0.01 \\
\hline . & -0.01 & 0.04 & 0.01 \\
\hline 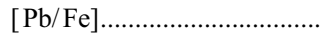 & 0.10 & -0.09 & -0.02 \\
\hline
\end{tabular}

show no evidence of a dependence on these light-element abundances. This decoupling of light-element abundances from that of Eu is confirmed by previous studies of well-sampled clusters. Several authors have compared [Eu/Fe] for globular clusters with results for Galactic field stars. The evidence is that the $[\mathrm{Eu} / \mathrm{Fe}]-$ $[\mathrm{Fe} / \mathrm{H}]$ trends (i.e., the apparent independence of $[\mathrm{Eu} / \mathrm{Fe}]$ on $[\mathrm{Fe} / \mathrm{H}]$ for $[\mathrm{Fe} / \mathrm{H}]<-0.7$ ) are the same for both samples (see, e.g., Gratton et al. 2004; James et al. 2004; Pritzl et al. 2005).

In summary, globular cluster and field stars appear with remarkably few exceptions and to within measurement errors to have the same $[\mathrm{Eu} / \mathrm{Fe}]$ ratio. Our measurements of this ratio for M4 and M5 are consistent with previous measurements for these clusters and the common cluster-field star set of data.

\subsubsection{The $[\mathrm{Y} / \mathrm{Fe}],[\mathrm{Zr} / \mathrm{Fe}],[\mathrm{Ba} / \mathrm{Fe}]$, and $[\mathrm{La} / \mathrm{Fe}]$ Ratios}

Among the few heavy elements in abundance analyses of globular cluster stars from which the $s$-process contribution may be assessed, $\mathrm{Ba}$ is the most widely reported. Other elements for which abundance data are available for several clusters include $\mathrm{Y}, \mathrm{Zr}$, and $\mathrm{La}$.

For most globular clusters, the $[\mathrm{Ba} / \mathrm{Fe}]$ ratio is positive $([\mathrm{Ba} / \mathrm{Fe}] \simeq+0.2)$ and independent of $[\mathrm{Fe} / \mathrm{H}]$ over the interval -0.7 to about -1.5 . For the few investigated clusters with $[\mathrm{Fe} / \mathrm{H}] \leq-1.8,[\mathrm{Ba} / \mathrm{Fe}]$ declines to roughly 0.0 at $[\mathrm{Fe} / \mathrm{H}]$ of -2.0 to -2.5 . This run with $[\mathrm{Fe} / \mathrm{H}]$ for the clusters is matched by the field stars (James et al. 2004). The spread in $[\mathrm{Ba} / \mathrm{Fe}]$ at a given $[\mathrm{Fe} / \mathrm{H}]$ is about \pm 0.2 dex, a value consistent with the errors of measurement, with several outliers. The sole outlier with $[\mathrm{Ba} / \mathrm{Fe}]$ above the mean trend is M4 with an excess of about $0.4 \mathrm{dex}$ in $[\mathrm{Ba} / \mathrm{Fe}]$. The outliers below the mean trend are NGC 3201, Rup 106, and Palomar 12 (Brown et al. 1997; Gonzalez \& Wallerstein 1998), as well as NGC 5694 (Lee et al. 2006). Data for $[\mathrm{La} / \mathrm{Fe}]$ is less extensive but suggests $[\mathrm{La} / \mathrm{Fe}] \simeq+0.1$ with M4 again as an outlier $([\mathrm{La} / \mathrm{Fe}]=+0.45)$ but with the Ba outliers NGC 3201 and Pal 12 as conformers to the mean value. NGC 5694 is again an outlier with $[\mathrm{La} / \mathrm{Fe}]=-0.26$ based on analysis of a single star (Lee et al. 2006). The [Y/Fe] ratio is close to zero (James et al. 2004). Our result for M5 at +0.2 from just two stars may be an (unlikely) outlier. At $[\mathrm{Y} / \mathrm{Fe}]=+0.7$, M4 is certainly an outlier.

The discussed data, as well as less extensive data on other heavy elements, show that the vast majority of the clusters are not distinguishable from field stars by their abundances of $\mathrm{Ba}$ and other heavy elements. For a given cluster, there is no compelling evidence for a star-to-star variation correlated with the variations seen among the light elements for many clusters. There are, however, examples of individual cluster stars with $s$-process

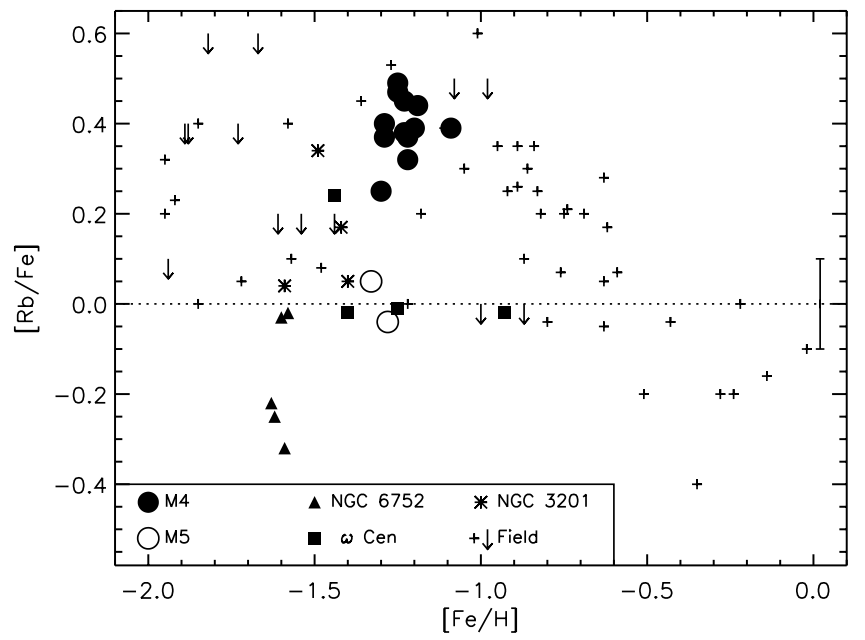

FIG. 3.- $[\mathrm{Rb} / \mathrm{Fe}]$ vs. $[\mathrm{Fe} / \mathrm{H}]$ for M4 ( filled circles) and M5 (open circles). Triangles show NGC 6752 (Yong et al. 2006b), squares represent $\omega$ Cen (Smith et al. 2000), asterisks show NGC 3201 (Gonzalez \& Wallerstein 1998), and plus signs and upper limits represent field stars from Tomkin \& Lambert (1999) and Gratton \& Sneden (1994). A representative error bar is shown. The abundances have been shifted onto the Smith et al. (2000) scale. [See the electronic edition of the Journal for a color version of this figure.]

enrichments, e.g., the $\mathrm{CH}$ stars in M2 and M55 (Smith \& Mateo 1990; Briley et al. 1993), the possible CH star in M22 (McClure \& Norris 1977; Vanture \& Wallerstein 1992), a Y-rich star in M13 (Cohen \& Meléndez 2005), and a hint of $\mathrm{Zr}$ abundance variations in 47 Tuc (Wylie et al. 2006), M5 (Ramírez \& Cohen 2003), and NGC 6752 (Yong et al. 2005). While M15 exhibits variations of $\mathrm{Ba}$ and $\mathrm{Eu}$, these variations are not correlated with the lightelement abundances, and the ratio $[\mathrm{Ba} / \mathrm{Eu}]$ is constant from star to star (Sneden et al. 1997; Otsuki et al. 2006).

\subsection{Rubidium}

In M4 the mean $\mathrm{Rb}$ abundance is $[\mathrm{Rb} / \mathrm{Fe}]=0.39 \pm 0.02(\sigma=$ $0.07)$ and in $\mathrm{M} 5$ the mean abundance is $[\mathrm{Rb} / \mathrm{Fe}]=0.00 \pm 0.05$ $(\sigma=0.06)$. As expected, M4 has a higher abundance of $\mathrm{Rb}$ than M5. While $50 \%$ of the solar $\mathrm{Rb}$ abundance can be attributed to the $r$-process (Burris et al. 2000), M4 has a slightly lower [Eu/Fe] ratio than does M5, and therefore it would be difficult to attribute any $\mathrm{Rb}$ excess in M4 relative to M5 as being due to a larger $r$-process contribution. Neither cluster shows any evidence for a dispersion in $\mathrm{Rb}$ abundances. That is, the scatter in the $\mathrm{Rb}$ abundances within M4 and M5 is small and can be attributed entirely to the measurement uncertainties. The $\mathrm{Rb}$ abundances are not correlated with $\mathrm{O}$ or $\mathrm{Na}$ (the two elements that exhibit large starto-star abundance variations).

The Rb abundances of M4 and M5 are compared with the limited data in the literature for other clusters and field stars. In Figure 3, we compare the $[\mathrm{Rb} / \mathrm{Fe}]$ abundance ratios with field dwarfs and giants (Gratton \& Sneden 1994; Tomkin \& Lambert 1999), as well as the globular clusters NGC 3201 (Gonzalez \& Wallerstein 1998), $\omega$ Cen (Smith et al. 2000), and NGC 6752 (Yong et al. 2006b). M5 has [Rb/Fe] ratios that are essentially identical to those of $\omega$ Cen giants at the same metallicity. (We note that $\omega$ Cen is now regarded as the nucleus of an accreted dwarf spheroidal galaxy [Smith et al. 2000].) These Rb abundances are comparable to the lowest values seen in field stars at the same metallicity. Given that M4 is s-process enriched relative to other globular clusters and field stars, it is puzzling that at the metallicity of M4, the majority of field stars share the same $[\mathrm{Rb} / \mathrm{Fe}]$ ratio. With the addition of M4 and M5, the [Rb/Fe] ratios in NGC 6752 

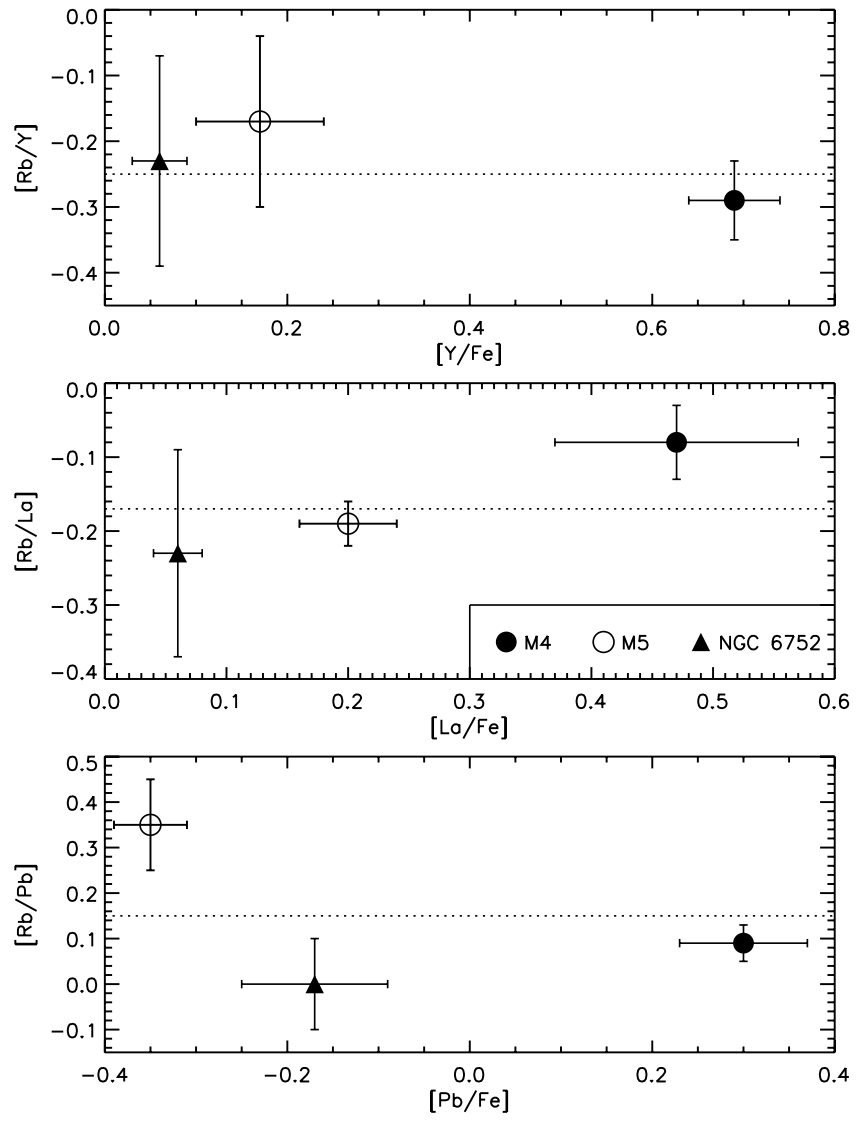

FIG. 4.- $[\mathrm{Rb} / \mathrm{Y}]$ vs. $[\mathrm{Y} / \mathrm{Fe}]$ (top), $[\mathrm{Rb} / \mathrm{La}]$ vs. [ $\mathrm{La} / \mathrm{Fe}]$ (middle), and $[\mathrm{Rb} / \mathrm{Pb}]$ vs. $[\mathrm{Pb} / \mathrm{Fe}]($ bottom) for M4, M5, and NGC 6752. In each panel, the dotted line represents the mean value.

appear rather low compared to other globular clusters and field stars. While the sample sizes remain small and the analyses have been performed by different investigators, the globular clusters cover a wider range of $[\mathrm{Rb} / \mathrm{Fe}]$ values than do the field stars in the same metallicity regime.

The relative abundances of $\mathrm{Rb}, \mathrm{Y}$, and $\mathrm{Zr}$ are very similar for M4 and M5. In Figure 4, the top panel shows [ $\mathrm{Rb} / \mathrm{Y}$ ] versus [ $\mathrm{Y} / \mathrm{Fe}]$ for mean values for M4, M5, and NGC 6752. The dotted line corresponds to $[\mathrm{Rb} / \mathrm{Y}]=-0.25$, the mean value for the three clusters. Published results for NGC 3201 and $\omega$ Cen do not fall on the line but straddle it. This may indicate zero-point differences between our and other analyses or a real cluster-to-cluster difference among the $\mathrm{Rb}, \mathrm{Y}$, and $\mathrm{Zr}$ abundances. Considering the individual measurements, we find $[\mathrm{Rb} / \mathrm{Y}]=-0.29 \pm 0.02(\sigma=$ $0.06)$ for $\mathrm{M} 4$ and $[\mathrm{Rb} / \mathrm{Y}]=-0.17 \pm 0.10(\sigma=0.13)$ for M5. For $\mathrm{Zr}$, we find $[\mathrm{Rb} / \mathrm{Zr}]=0.17 \pm 0.03(\sigma=0.08)$ for $\mathrm{M} 4$ and $[\mathrm{Rb} / \mathrm{Zr}]=0.08 \pm 0.08(\sigma=0.11)$ for M5. (These $\mathrm{Zr}$ abundances have been shifted by +0.3 dex onto the Smith et al. [2000] scale; see Yong et al. [2006b] for details.) Neither the [Rb/Y] nor the $[\mathrm{Rb} / \mathrm{Zr}]$ ratio exhibits a star-to-star dispersion, and neither is correlated with $\mathrm{O}$ or $\mathrm{Na}$ abundances. In Figure 5 we compare the $[\mathrm{Rb} / \mathrm{Zr}]$ abundance ratio with field dwarfs and giants, as well as globular cluster giants. Compared to the $\omega$ Cen giants at a similar metallicity, M4 and M5 have [Rb/Zr] ratios 0.4-0.5 dex higher (which may be due to systematic offsets). For M4, the difference arises due to $\omega$ Cen having less $\mathrm{Rb}$ and more Zr. For M5, the difference results from $\omega$ Cen having similar $\mathrm{Rb}$ and considerably more $\mathrm{Zr}$.

The similarity in heavy-element abundance ratios involving $\mathrm{Rb}$ extends to $[\mathrm{Rb} / \mathrm{La}]$ - see Figure 4 (middle). The indication from

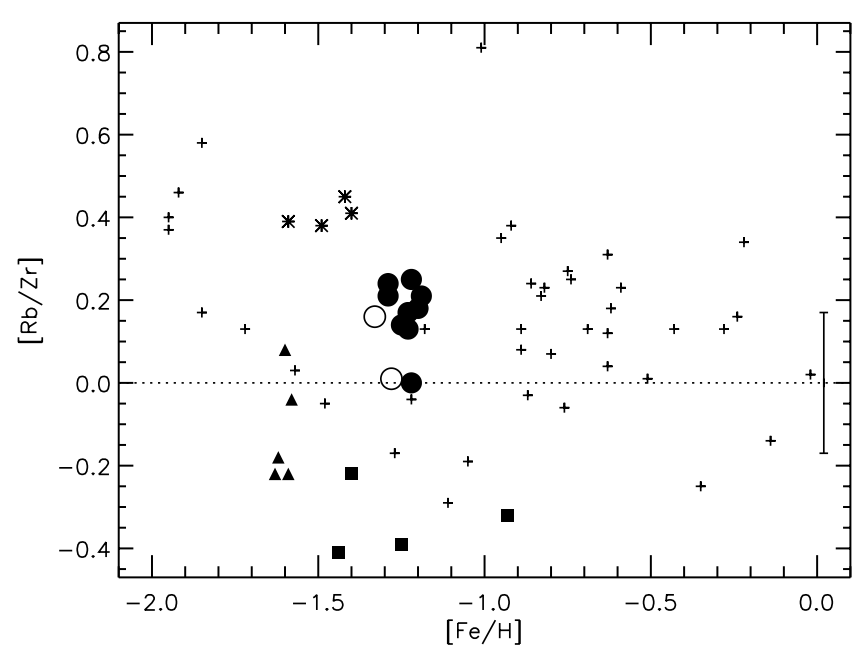

FIG. 5.- $[\mathrm{Rb} / \mathrm{Zr}]$ vs. [Fe/H $]$. The symbols are the same as in Fig. 3. The abundances have been shifted onto the Smith et al. (2000) scale. A representative error bar is shown. [See the electronic edition of the Journal for a color version of this figure.]

our analyses is that the relative abundances of $\mathrm{Rb}$ to $\mathrm{Y}, \mathrm{Zr}$, and $\mathrm{La}$ are the same for M5 and NGC 6752, as well as M4, the cluster clearly enriched in $s$-process elements. This demonstrates that the source of the M4 enrichment is identical to that providing the $s$-process elements for the natal material for M5 and NGC 6752. The abundance of $\mathrm{Rb}$ relative to the other elements is slightly subsolar, i.e., $[\mathrm{Rb} / \mathrm{Y}]=-0.25$. There is a well-known difference between the spectroscopically determined $\mathrm{Rb}$ abundance and that obtained from carbonaceous meteorites: $\log \epsilon(\mathrm{Rb})=2.60$ versus 2.33, respectively (Asplund et al. 2005). If the meteoritic value is adopted, $[\mathrm{Rb} / \mathrm{Y}]$ and similar ratios are increased by 0.27 dex and rendered indistinguishable from the solar ratios. Then, $s$-process donors to the natal clouds of the globular clusters and of local stars including the Sun must have been similar.

\subsection{Lead}

In M4 the mean $\mathrm{Pb}$ abundance is $[\mathrm{Pb} / \mathrm{Fe}]=0.30 \pm 0.02(\sigma=$ $0.07)$, and in M5 the mean abundance is $[\mathrm{Pb} / \mathrm{Fe}]=-0.35 \pm$ $0.02(\sigma=0.04)$. We note that $20 \%$ of the solar $\mathrm{Pb}$ abundance is attributable to the $r$-process (Burris et al. 2000), but the [Eu/Fe] ratio is comparable for M4 and M5. Again, since M4 has a lower $[\mathrm{Eu} / \mathrm{Fe}]$ ratio than $\mathrm{M} 5$, the excess $\mathrm{Pb}$ in $\mathrm{M} 4$ relative to $\mathrm{M} 5$ cannot be due to an increased $r$-process contribution for M4. The excess likely arises from more complete processing down the $s$-process chain to its termination at $\mathrm{Pb}$. The difference in $[\mathrm{Pb} / \mathrm{Fe}]$ between M4 and M5 is 0.65 dex. For the other neutron-capture elements synthesized via the $s$-process, the difference in $[\mathrm{X} / \mathrm{Fe}]$ between M4 and M5 is typically 0.4 dex. Regarding the scatter in Pb abundances in M4 and M5, we again find that the small dispersion can be entirely explained by the measurement uncertainties. The $\mathrm{Pb}$ abundances in M4 and M5 are not correlated with $\mathrm{O}$ or $\mathrm{Na}$.

In Figure 6 we compare the $[\mathrm{Pb} / \mathrm{Fe}]$ abundance ratios with field stars (Sneden et al. 1998; Travaglio et al. 2001), as well as the globular clusters M13 and NGC 6752 (Yong et al. 2006b). Data on $\mathrm{Pb}$ in the field stars is obviously very limited. The one certain data point is for HD 23439A, a star enriched in $s$-process products (Tomkin \& Lambert 1999), with $[\mathrm{Pb} / \mathrm{Fe}]=+0.6$. The other three data points not indicated as upper limits in Figure 6 are marked with the customary “:” by Travaglio et al. (2001), and inspection of their published spectra shows that an alternative designation as an upper limit is possibly more appropriate. Our $\mathrm{Pb}$ abundance determination for HD 141531 (Yong et al. 2006b) is consistent 


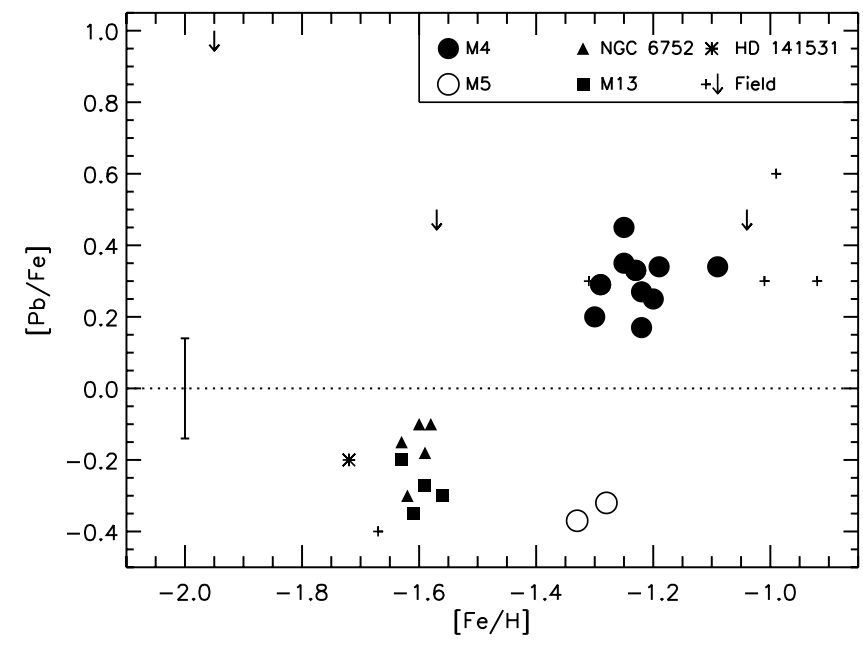

FIG. 6.- $[\mathrm{Pb} / \mathrm{Fe}]$ vs. $[\mathrm{Fe} / \mathrm{H}]$ for M4 (filled circles) and M5 (open circles). Triangles show NGC 6752, squares represent M13, and the asterisk shows HD 141531 (Yong et al. 2006b). The plus signs and upper limits represent field stars from Sneden et al. (1998) and Travaglio et al. (2001). A representative error bar is shown. [See the electronic edition of the Journal for a color version of this figure.]

with the subsolar $[\mathrm{Pb} / \mathrm{Fe}]$ ratios for NGC 6752, M5, and M13. Although more $\mathrm{Pb}$ abundances are needed for field stars, we suppose that the $\mathrm{Pb}$ abundances of the globular clusters NGC 6752, M5, and M13 and normal field stars are in fair agreement, as found for $\mathrm{Rb}, \mathrm{Y}, \mathrm{Zr}, \mathrm{Ba}$, and other heavy elements.

In Figure 4 (bottom) we plot [ $\mathrm{Rb} / \mathrm{Pb}$ ] versus [ $\mathrm{Pb} / \mathrm{Fe}]$ for $\mathrm{M} 4$, $\mathrm{M} 5$, and NGC 6752 . Interestingly, the $[\mathrm{Rb} / \mathrm{Pb}]$ ratios for $\mathrm{M} 4$ and NGC 6752 appear similar, which again suggests that the source of the $s$-process enrichment in M4 may be identical to the source of the $s$-process elements for NGC 6752. However, in this figure M5 now appears to be the outlier.

Given the small comparison sample, clearly it would be of great interest to expand the measurements of $\mathrm{Pb}$ to larger samples of field stars. The synthetic spectra in this study and in Yong et al. (2006b) indicate that measurements of $\mathrm{Pb}$ in cool giants in the range $-2.0 \leq[\mathrm{Fe} / \mathrm{H}] \leq-1.0$ are feasible.

\subsection{Consequences for the Primordial Scenario}

Formation of the Galactic and extragalactic globular clusters remains a subject with many unanswered questions (Brodie \& Strader 2006). From the point of view of the quantitative spectroscopist interested in the composition of stars in the Galactic globular clusters, two questions seem preeminent: (1) Why do the cluster and field stars follow the same $[\mathrm{X} / \mathrm{Fe}]$ versus $[\mathrm{Fe} / \mathrm{H}]$ relations? (2) What causes the light-element abundance variations first observed among cluster giant stars but now found also in subgiant and main-sequence stars?

The simplest answer to question 1 would appear to be that the clusters are formed during episodic mergers in the hierarchical formation of the Galaxy, as discussed by Bekki et al. (2002) and Beasley et al. (2002). If the gas content of the protocluster is dominated by that from the Galaxy and not the infalling system, the composition of the cluster stars will be very similar to that of the Galactic stars formed just preceding the merger. Gas remaining after star formation in the cluster will be ejected by supernovae from the massive stars. Star formation will be restricted to a single generation of stars, i.e., stars within a given cluster will have the identical metallicity. Indeed, the complete composition of all stars will be the same but for changes resulting from stellar evolution and the agents responsible for the star-to-star variations among light-element abundances. These agents comprise what is widely referred to as "the primordial scenario."

For the majority of the Galactic globular clusters subjected to abundance analysis, the compositions do closely resemble field stars of the cluster's metallicity. This is as expected on the merger hypothesis. M4 is an apparent exception with its overabundance of heavy elements (Y, Zr, Ba, La, etc., but not $\mathrm{Eu}$ ) matched by very few field stars. Some of the field stars with the cluster's degree of overabundance of $s$-process products are binaries in which the products were transferred to the present star when its companion was an AGB star. There are very few known examples where mass transfer across a binary is not a viable explanation. The case of HD 23439A and B ( Tomkin \& Lambert 1999) would appear to be such a case. HD 23439A shows no sign of radial velocity variation during $14 \mathrm{yr}$ of monitoring (Latham et al. 2002). On the other hand, HD 23439B is a binary, but its period and orbital eccentricity do not suggest mass transfer (Latham et al. 2002).

Other clusters with few counterparts among field stars include Rup 106 and Pal 12. In their case, the Sagittarius dwarf spheroidal galaxy now undergoing destruction by the Galaxy has giants with the peculiar composition of Rup 106 and Pal 12 (Sbordone et al. 2007), indicating that they belong to the dwarf spheroidal galaxy. A conjecture is that the cluster M4 was captured from a stellar system in which chemical evolution had led to $s$-process enrichment; a change in the IMF might have been immediately responsible. However, consideration of space velocities reveals that M4's orbit is restricted to the inner disk and bulge (apocentric radius, $R_{a}=5.9 \mathrm{kpc}$ ), whereas M5's orbit may be more consistent with a capture event, $R_{a}=35.4 \mathrm{kpc}$ (Dinescu et al. 1999). Given M4's orbit, a comparison of the $s$-process elements in M4 and comparable metallicity stars of the inner disk and bulge would be of great interest.

In the primordial scenario, the star-to-star abundance variations apart from those attributable to dredge-up in giants arise from the accretion of gas (or contamination of natal clouds) to varying degrees by cluster stars. The accreted gas is assumed to be mass lost from the more massive and now dead stars of the cluster. Proposed candidates include IM-AGBs who experience extensive nucleosynthesis and lose their envelopes at low velocity so that the ejecta reside within the cluster's potential (Cottrell \& Da Costa 1981). Recently, a role for massive stars in polluting the cluster's environment has been proposed (Decressin et al. 2007; Smith 2006; Prantzos \& Charbonnel 2006).

Both M4 and M5 exhibit star-to-star abundance variations for the light elements, as does every well-studied Galactic globular cluster. The variations involve $\mathrm{O}, \mathrm{Na}, \mathrm{Mg}$, and $\mathrm{Al}$, as well as $\mathrm{C}$ and $\mathrm{N}$, for which there is a component related to dredge-up by giants. For the majority of the analyzed clusters (M4 is an obvious exception), the elemental abundances identified with the unpolluted cluster stars match those of field stars of the same metallicity. Two clusters have been studied for variations among the isotopic ratios of $\mathrm{Mg}$, but the normal stars in M13 and NGC 6752 (i.e., cluster stars with high $\mathrm{O}$, high $\mathrm{Mg}$, low $\mathrm{Na}$, low $\mathrm{Al}$, and whose $\mathrm{O}-\mathrm{Al}$ abundances match field stars at the same metallicity) had $\mathrm{Mg}$ isotope ratios that exceeded measurements in comparable metallicity field stars (Yong et al. 2003a, 2003b, 2006a).

In this study, we extended the elemental abundance measurements of $\mathrm{Rb}$ and $\mathrm{Pb}$ to the globular clusters M4 and M5. All four clusters that we have studied (M4, M5, M13, and NGC 6752) show light element variations, but constant $\mathrm{Rb}$ and $\mathrm{Pb}$ abundances within each cluster. Thus, the accreted gas providing the light element variations cannot contain significant amounts (or significant deficiencies) of $\mathrm{Rb}$ and $\mathrm{Pb}$. The accreted gas 
must have the same proportions of $\mathrm{Rb}$ and $\mathrm{Pb}$ as the ambient material.

The source of the gas may be IM-AGBs that at least qualitatively account for the range of abundance variations. A quantitative test is impossible at present because the predicted yields from metal-poor IM-AGBs may be model dependent (Busso et al. 2001; Ventura \& D'Antona 2005a, 2005b, 2005c). As far as $s$-products are concerned which, as we have emphasized, do not show abundance variations, IM-AGBs remain a viable candidate because Lattanzio et al. (2004) and O. Straniero (2006, private communication) suggest that IM-AGBs will not produce any $s$-process elements, since the mass of the He shell and the duration of the thermal pulse decreases with increasing AGB mass.

Massive stars achieve limited synthesis of $s$-process products but are held to be the sites of the weak $s$-process (and the $r$-process) contributing nuclides from the iron group up to about $\mathrm{Rb}, \mathrm{Y}$, and $\mathrm{Zr}$ (Raiteri et al. 1991). Predicted yields are such that massive stars could serve to provide the light-element abundance variations without leading to predicted variations for $\mathrm{Rb}, \mathrm{Y}$, and $\mathrm{Zr}$.

In short, the lack of abundance variations for $\mathrm{Rb}, \mathrm{Y}, \mathrm{Zr}$, and other $s$-process elements may not exclude either IM-AGBs or massive stars as sources of the accreted pollutants.

\section{CONCLUDING REMARKS}

In this paper we present measurements of the neutron-capture elements $\mathrm{Rb}$ and $\mathrm{Pb}$ in the globular clusters M4 and M5. While both clusters exhibit star-to-star abundance variations for the light elements, we find that the abundances of $\mathrm{Rb}$ and $\mathrm{Pb}$ are constant. None of the abundance ratios $[\mathrm{Rb} / \mathrm{Fe}],[\mathrm{Rb} / \mathrm{Zr}]$, and $[\mathrm{Pb} / \mathrm{Fe}]$ are correlated with $\mathrm{O}$ or $\mathrm{Na}$ abundances. In the primordial scenario, the abundance variations for the light elements are attributed to different levels of accretion of ejecta from IM-AGBs or massive stars. The fact that the heavy elements including $\mathrm{Rb}$ and $\mathrm{Pb}$ do not show abundance variations implies that the accreted material has the same composition as the ambient material for the heavy elements (i.e., the accreted material cannot be highly underabundant or overabundant in these elements). That the ratios $[\mathrm{Rb} / \mathrm{X}]$ for $\mathrm{X}=\mathrm{Y}, \mathrm{Zr}$, La are similar for M4 and M5 suggests that the sources of the $s$-process elements are similar and that M4 had a greater concentration of these products.

There remains a need to pursue additional observational tests of the primordial scenario. In particular, present data on the $\mathrm{Rb}$ and $\mathrm{Pb}$ abundances in field and cluster stars are sparse. The indication that the $\mathrm{Mg}$ isotopic ratios of unpolluted or normal cluster stars differ from those of field stars of the same metallicity deserves closer scrutiny by, in particular, extending the measurement of these isotopic ratios to additional clusters.

This research has made use of the SIMBAD database, operated at CDS, Strasbourg, France, and NASA's Astrophysics Data System. We thank the anonymous referee for helpful comments and Inese Ivans for sending observed and synthetic spectra. D. Y. thanks Amanda Karakas, Francesca D'Antona, Inese Ivans, John Lattanzio, Oscar Straniero, Paolo Ventura, and Roberto Gallino for helpful discussions and Chris Sneden for providing a line list for the $\mathrm{Pb}$ region. This research was performed while $\mathrm{D}$. B. P. held a National Research Council Research Associateship Award at NASA's Goddard Space Flight Center. D. L. L. acknowledges support from the Robert A. Welch Foundation of Houston, Texas. B. W. C. acknowledges support from the National Science Foundation through grant AST 03-05431 to the University of North Carolina. This research was supported in part by NASA through the American Astronomical Society's Small Research Grant Program.

\section{REFERENCES}

Abia, C., Busso, M., Gallino, R., Domínguez, I., Straniero, O., \& Isern, J. 2001, ApJ, 559, 1117

Allende Prieto, C., Lambert, D. L., \& Asplund, M. 2001, ApJ, 556, L63

Aoki, W., Ryan, S. G., Norris, J. E., Beers, T. C., Ando, H., \& Tsangarides, S. 2002, ApJ, 580, 1149

Arp, H. 1962, ApJ, 135, 311

Asplund, M., Grevesse, N., \& Sauval, A. J. 2005, in ASP Conf. Ser. 336, Cosmic Abundances as Records of Stellar Evolution and Nucleosynthesis, ed. T. G. Barnes III \& F. N. Bash (San Francisco: ASP), 25

Beasley, M. A., Baugh, C. M., Forbes, D. A., Sharples, R. M., \& Frenk, C. S. 2002, MNRAS, 333, 383

Bekki, K., Forbes, D. A., Beasley, M. A., \& Couch, W. J. 2002, MNRAS, 335, 1176

Bernstein, R., Shectman, S. A., Gunnels, S. M., Mochnacki, S., \& Athey, A. E. 2003, Proc. SPIE, 4841, 1694

Biémont, E., Baudoux, M., Kurucz, R. L., Ansbacher, W., \& Pinnington, E. H. 1991, A\&A, 249, 539

Blackwell, D. E., Lynas-Gray, A. E., \& Smith, G. 1995, A\&A, 296, 217

Briley, M. M., Smith, G. H., Hesser, J. E., \& Bell, R. A. 1993, AJ, 106, 142

Briley, M. M., Smith, V. V., Suntzeff, N. B., Lambert, D. L., Bell, R. A., \& Hesser, J. E. 1996, Nature, 383, 604

Brodie, J. P., \& Strader, J. 2006, ARA\&A, 44, 193

Brown, J. A., Wallerstein, G., \& Zucker, D. 1997, AJ, 114, 180

Burris, D. L., Pilachowski, C. A., Armandroff, T. E., Sneden, C., Cowan, J. J., \& Roe, H. 2000, ApJ, 544, 302

Busso, M., Gallino, R., Lambert, D. L., Travaglio, C., \& Smith, V. V. 2001, ApJ, 557, 802

Busso, M., Gallino, R., \& Wasserburg, G. J. 1999, ARA\&A, 37, 239

Cavallo, R. M., Suntzeff, N. B., \& Pilachowski, C. A. 2004, AJ, 127, 3411

Charbonnel, C. 1995, ApJ, 453, L41

Cohen, J. G., \& Meléndez, J. 2005, AJ, 129, 303

Cottrell, P. L., \& Da Costa, G. S. 1981, ApJ, 245, L79

Cowan, J. J., \& Sneden, C. 2006, Nature, 440, 1151

Decressin, T., Meynet, G., Charbonnel, C., Prantzos, N., \& Ekström, S. 2007, A\&A, 464, 1029

Demarque, P., Woo, J.-H., Kim, Y.-C., \& Yi, S. K. 2004, ApJS, 155, 667

Dinescu, D. I., Girard, T. M., \& van Altena, W. F. 1999, AJ, 117, 1792

Fenner, Y., Campbell, S., Karakas, A. I., Lattanzio, J. C., \& Gibson, B. K. 2004, MNRAS, 353, 789

Gonzalez, G., \& Wallerstein, G. 1998, AJ, 116, 765

Goriely, S., \& Siess, L. 2001, A\&A, 378, L25

Gratton, R. G., Bragaglia, A., Carretta, E., Clementini, G., Desidera, S., Grundahl, F., \& Lucatello, S. 2003, A\&A, 408, 529

Gratton, R. G., \& Sneden, C. 1994, A\&A, 287, 927

Gratton, R., Sneden, C., \& Carretta, E. 2004, ARA\&A, 42, 385

Gratton, R. G., et al. 2001, A\&A, 369, 87

Grevesse, N., \& Sauval, A. J. 1998, Space Sci. Rev., 85, 161

Grundahl, F., Briley, M., Nissen, P. E., \& Feltzing, S. 2002, A\&A, 385, L14

Ivans, I. I., Kraft, R. P., Sneden, C., Smith, G. H., Rich, R. M., \& Shetrone, M. 2001, AJ, 122, 1438

Ivans, I. I., Sneden, C., Kraft, R. P., Suntzeff, N. B., Smith, V. V., Langer, G. E., \& Fulbright, J. P. 1999, AJ, 118, 1273

James, G., François, P., Bonifacio, P., Carretta, E., Gratton, R. G., \& Spite, F. 2004, A\&A, 427, 825

Karakas, A. I., \& Lattanzio, J. C. 2003, Publ. Astron. Soc. Australia, 20, 279 Kraft, R. P. 1994, PASP, 106, 553

Kurucz, R. 1993, CD-ROM 13, ATLAS9 Stellar Atmosphere Programs and 2 km/s Grid (Cambridge: SAO)

Kurucz, R. L., Furenlid, I., \& Brault, J. 1984, Solar Flux Atlas from 296 to 1300 NM (Sunspot: NSO)

Lambert, D. L., Heath, J. E., Lemke, M., \& Drake, J. 1996, ApJS, 103, 183

Lambert, D. L., \& Luck, R. E. 1976, Observatory, 96, 100

Lambert, D. L., Smith, V. V., Busso, M., Gallino, R., \& Straniero, O. 1995, ApJ, 450, 302

Latham, D. W., Stefanik, R. P., Torres, G., Davis, R. J., Mazeh, T., Carney, B. W., Laird, J. B., \& Morse, J. A. 2002, AJ, 124, 1144

Lattanzio, J., Karakas, A., Campbell, S., Elliott, L., \& Chieffi, A. 2004, Mem. Soc. Astron. Italiana, 75, 322

Lattanzio, J. C., \& Tout, C. A. 2006, EAS Pub. Ser., 19, 189

Lawler, J. E., Bonvallet, G., \& Sneden, C. 2001a, ApJ, 556, 452 
Lawler, J. E., Wickliffe, M. E., den Hartog, E. A., \& Sneden, C. 2001b, ApJ, 563,1075

Lee, J.-W., López-Morales, M., \& Carney, B. W. 2006, ApJ, 646, L119

Lee, S. W. 1977, A\&AS, 27, 367

McClure, R. D., \& Norris, J. 1977, ApJ, 217, L101

Norris, J. 1981, ApJ, 248, 177

Otsuki, K., Honda, S., Aoki, W., Kajino, T., \& Mathews, G. J. 2006, ApJ, 641, L117

Prantzos, N., \& Charbonnel, C. 2006, A\&A, 458, 135

Pritzl, B. J., Venn, K. A., \& Irwin, M. 2005, AJ, 130, 2140

Raiteri, C. M., Busso, M., Picchio, G., \& Gallino, R. 1991, ApJ, 371, 665

Ramírez, S. V., \& Cohen, J. G. 2003, AJ, 125, 224

Renzini, A., \& Fusi Pecci, F. 1988, ARA\&A, 26, 199

Sbordone, L., Bonifacio, P., Buonanno, R., Marconi, G., Monaco, L., \& Zaggia, S. 2007, A\&A, 465, 815

Smith, G. H. 1987, PASP, 99, 67 2006, PASP, 118, 1225

Smith, G. H., \& Mateo, M. 1990, ApJ, 353, 533

Smith, G. H., \& Norris, J. 1983, ApJ, 264, 215

Smith, G. H., Shetrone, M. D., Briley, M. M., Churchill, C. W., \& Bell, R. A. 1997, PASP, 109, 236

Smith, V. V., Suntzeff, N. B., Cunha, K., Gallino, R., Busso, M., Lambert, D. L., \& Straniero, O. 2000, AJ, 119, 1239
Sneden, C. 1973, ApJ, 184, 839

Sneden, C., Cowan, J. J., Burris, D. L., \& Truran, J. W. 1998, ApJ, 496, 235

Sneden, C., Kraft, R. P., Shetrone, M. D., Smith, G. H., Langer, G. E., \& Prosser, C. F. 1997, AJ, 114, 1964

Suntzeff, N. B., \& Smith, V. V. 1991, ApJ, 381, 160

Sweigart, A. V., \& Mengel, J. G. 1979, ApJ, 229, 624

Tomkin, J., \& Lambert, D. L. 1983, ApJ, 273, 722 1999, ApJ, 523, 234

Travaglio, C., Gallino, R., Busso, M., \& Gratton, R. 2001, ApJ, 549, 346

Vanture, A. D., \& Wallerstein, G. 1992, PASP, 104, 888

Ventura, P., \& D’Antona, F. 2005a, A\&A, 431, 279 2005b, A\&A, 439, 1075 2005c, ApJ, 635, L149

Wylie, E. C., Cottrell, P. L., Sneden, C. A., \& Lattanzio, J. C. 2006, ApJ, 649, 248

Yong, D., Aoki, W., \& Lambert, D. L. 2006a, ApJ, 638, 1018

Yong, D., Aoki, W., Lambert, D. L., \& Paulson, D. B. 2006b, ApJ, 639, 918

Yong, D., Grundahl, F., Lambert, D. L., Nissen, P. E., \& Shetrone, M. D. 2003a, A\&A, 402, 985

Yong, D., Grundahl, F., Nissen, P. E., Jensen, H. R., \& Lambert, D. L. 2005, A\&A, 438, 875

Yong, D., Lambert, D. L., \& Ivans, I. I. 2003b, ApJ, 599, 1357 